\title{
Extracting a stroke phenotype risk factor from Veteran Health Administration clinical reports: an information content analysis
}

Danielle L. Mowery ${ }^{1,2^{*}}$, Brian E. Chapman ${ }^{1,2}$, Mike Conway ${ }^{1}$, Brett R. South ${ }^{1,2}$, Erin Madden ${ }^{3}$, Salomeh Keyhani ${ }^{3}$ and Wendy W. Chapman ${ }^{1,2}$

\begin{abstract}
Background: In the United States, 795,000 people suffer strokes each year; 10-15\% of these strokes can be attributed to stenosis caused by plaque in the carotid artery, a major stroke phenotype risk factor. Studies comparing treatments for the management of asymptomatic carotid stenosis are challenging for at least two reasons: 1) administrative billing codes (i.e., Current Procedural Terminology (CPT) codes) that identify carotid images do not denote which neurovascular arteries are affected and 2) the majority of the image reports are negative for carotid stenosis. Studies that rely on manual chart abstraction can be labor-intensive, expensive, and time-consuming. Natural Language Processing (NLP) can expedite the process of manual chart abstraction by automatically filtering reports with no/insignificant carotid stenosis findings and flagging reports with significant carotid stenosis findings; thus, potentially reducing effort, costs, and time.
\end{abstract}

Methods: In this pilot study, we conducted an information content analysis of carotid stenosis mentions in terms of their report location (Sections), report formats (structures) and linguistic descriptions (expressions) from Veteran Health Administration free-text reports. We assessed an NLP algorithm, pyConText's, ability to discern reports with significant carotid stenosis findings from reports with no/insignificant carotid stenosis findings given these three document composition factors for two report types: radiology (RAD) and text integration utility (TIU) notes.

Results: We observed that most carotid mentions are recorded in prose using categorical expressions, within the Findings and Impression sections for RAD reports and within neither of these designated sections for TIU notes. For RAD reports, pyConText performed with high sensitivity $(88 \%)$, specificity $(84 \%)$, and negative predictive value (95\%) and reasonable positive predictive value (70 \%). For TIU notes, pyConText performed with high specificity $(87 \%)$ and negative predictive value $(92 \%)$, reasonable sensitivity $(73 \%)$, and moderate positive predictive value (58\%). pyConText performed with the highest sensitivity processing the full report rather than the Findings or Impressions independently.

Conclusion: We conclude that pyConText can reduce chart review efforts by filtering reports with no/insignificant carotid stenosis findings and flagging reports with significant carotid stenosis findings from the Veteran Health Administration electronic health record, and hence has utility for expediting a comparative effectiveness study of treatment strategies for stroke prevention.

Keywords: Natural language processing, Stroke, Phenotype, Information extraction

\footnotetext{
* Correspondence: danielle.mowery@utah.edu

${ }^{1}$ Department of Biomedical Informatics, University of Utah, Salt Lake City, UT, USA

${ }^{2}$ IDEAS Center, Veteran Affair Health Care System, Salt Lake City, UT, USA

Full list of author information is available at the end of the article
} 


\section{Background}

In biomedicine, we define a disease or mutant phenotype experienced by an individual as observations caused by interactions between the environment and his/her genome that differ from the expected, "normal" wild type. Over the last several years, the biomedical community has begun to leverage informatics and electronic health record (EHR) data to define and identify phenotypes for genetic analyses using genome-wide (GWAS) and phenotype-wide (PheWAS) association studies [1, 2]. For instance, PheKB is a knowledgebase that contains phenotypes defined using EHR data and subsequently validated within one or more institutions. This catalogue of phenotypes was primarily generated by the Electronic Medical Records and Genomics (eMERGE) network, a United States (US) National Human Genome Research Institute-funded consortium, but is also supplemented by the informatics community at large (https://phekb.org/ phenotypes) [3-5]. Similarly, the Strategic Health IT Research Program for secondary use of EHRs (SHARPn), funded by the US Office of the National Coordinator for Health Information Technology, aims to transform heterogeneous EHR data from various sites into a standardized form to support high-throughput phenotyping [6].

\section{Phenotyping with electronic health record data}

Several phenotypes have been the foci of informatics studies including cancer, diabetes, heart failure, rheumatoid arthritis, drug side effects, cataract, pneumonia, asthma, peripheral artery disease, and hypertension [7]. EHRs provide a groundbreaking opportunity to define and identify these complex phenotypes leveraging data elements from the longitudinal patient record. Specifically, patient phenotypes are often inferred from both structured EHR data elements (e.g., administrative billing codes, vital signs, medications, laboratory values from data fields including dropdown lists and checkboxes) and unstructured EHR data elements (e.g., symptoms, signs, histories, and diagnoses within clinical notes including progress notes and discharge summaries). These heterogeneous data elements are then mapped to logical representations used to classify a patient into one or more phenotypes [8]. Outstanding challenges remain for next-generation phenotyping of EHR data including the need for approaches that address data complexity, inaccuracy, coverage, and biases [9].

\section{Natural language processing}

Traditionally, International Classification of Disease (ICD-9) billing codes have been leveraged to identify phenotype risk factors with variable results. Inaccurate performance can result from poor granularity within code descriptions and documentation of risk factors in patient clinical texts $[10,11]$. Natural language processing (NLP) may improve risk factor detection by identifying missed risk factor mentions (improving sensitivity) and filtering spurious risk factor mentions (improving positive predictive value) from these clinical texts. However, extracting risk factors associated with phenotypes from clinical texts can be challenging due to the usage of variable lexical expressions (e.g., "occlusion", "reduced arterial diameters"), ambiguous abbreviations (PAD can stand for "peripheral artery disease" or "pain and distress"), spelling errors ("diabetes" misspelled as "diabeetes"), and telegraphic constructions (e.g., "PHx: HTN" means "past history of hypertension") within clinical texts. Furthermore, multiple mentions of the same risk factor can be recorded within and across reports. This information might be integrated with structured data elements requiring logic to classify a patient with a phenotype. The success of an algorithm is often defined by performance metrics of sensitivity (or recall), positive predictive value (or precision), negative predictive value, and specificity by comparing the predicted phenotype from the system/algorithm against the coded phenotype from a domain expert [12].

\section{Extracting stroke risk factors using natural language processing}

NLP has been applied and, at times, integrated with structured data to successfully identify several stroke risk factors such as peripheral artery disease [5, 13], diabetes $[4,14]$, heart failure [15], and hypertension [16] as part of large, coordinated research projects. Specifically, Savova et al. extended the Clinical Text Analysis and Knowledge Extraction System to extract and classify positive, negative, probable, and unknown mentions of peripheral artery disease (PAD) [13]. Kullo et al. then leveraged this system to encode case-control status, comorbidities, and cardiovascular risk factors from the EHR for a GWAS study of PAD cases and controls for the eMERGE project [5]. Wilke et al. applied the FreePharma system to extract medication histories and combine them with diagnoses and laboratory results to identify a diabetes mellitus cohort as part of the Marshfield Clinic Personalized Medicine Research Project (PMRP) [14]. Kho et al. extracted diagnoses, medications, and laboratory results leveraging NLP to encode variables from unstructured fields for various sites to identify type 2 diabetes cases and controls for a multi-institutional GWAS study also as part of the eMERGE project [4]. Garvin et al. extracted left ventricular ejection fraction as an indicator for heart failure using the Unstructured Information Management Architecture (UIMA) as part of a Translational Use Case Project and quality improvement project within the Veteran Affairs (VA) Consortium for Healthcare Informatics Research (CHIR) [15]. Finally, Thompson et al. translated the nine algorithms 
for phenotypes including hypertension developed from the eMERGE project into the Quality Data Model (QDM) to support EHR-based quality measures [16].

Although NLP has addressed many stroke-associated risk factors for genotype-phenotype and other studies, few studies have leveraged NLP to identify these risk factors specifically for stroke prevention research. Furthermore, to our knowledge, no NLP study has targeted significant carotid stenosis - a known risk factor for stroke. Our long-term goal is to develop a comprehensive stroke phenotyping framework that extracts predictors of stroke subtypes e.g., ischemic or hemorrhagic as well as their precise endotypes e.g., ischemic stroke endotypes of cardiac embolism, large artery atherosclerosis, or lacunar infarction, other uncommon causes, from the EHR powered by NLP. Our short-term goal is to develop an NLP algorithm for a National Institute of Health (NIH)-sponsored comparative effectiveness study of ischemic stroke prevention treatments that automatically filters carotid reports for patients exhibiting no/ insignificant carotid stenosis of the internal or common carotid arteries from chart review. In this pilot study, we completed a qualitative and quantitative study of where and how mentions of carotid stenosis findings occur in radiology reports and how this affects an NLP algorithm's performance.

\section{Methods}

In this Institute Review Board (IRB or Ethics committee) and Veteran Affairs (VA) approved pilot study, we aimed to conduct an information content analysis of a major predictor of stroke, significant stenosis of the internal or common carotid arteries, for a sample of free-text reports from the Veteran Health Administration. Our goal is to automatically distinguish reports denoting one or more sides of significant stenosis (defined as greater than $50 \%$, moderate, or severe stenosis) from reports denoting no/insignificant stenosis (defined as negated, ruled out, mild, less than $50 \%$ stenosis) from both of the internal or common carotid arteries. In this study, we conducted an information content analysis of carotid stenosis findings with respect to three aspects of document composition - location (Sections), format (structures), and descriptions (expressions). We assessed the performance of pyConText, an NLP algorithm, at automatically extracting and encoding stenosis findings given these three document constituents.

\section{Dataset}

We selected all reports from the VA EHR for patients with an administratively documented carotid image procedure code (CPT code) restricted to those within -1 to +9 days of the procedure code date and that contained a carotid term ("carot", "ica", "lica", "rica", or "cca"). In our previous study, we leveraged 418 randomly sampled VA radiology reports for developing our NLP algorithm, pyConText, to identify mention-level stenosis findings [17]. We extended this previous study by randomly selecting a new set of reports to classify document-level stenosis based on identified mention-level carotid stenosis findings. This dataset consists of 598 radiology reports (RAD: mainly ultrasound reports) and 598 text integration utility notes (TIU: mainly progress notes, carotid duplex exams, and carotid triplex exams) (see Fig. 1). Because much of our algorithm development was completed during our previous study $[17,18]$ and the prevalence of stenosis positive reports is low, we chose a larger testing set for each report type. We also chose to maintain the natural distribution to give us a better sense of whether pyConText could correctly retain stenosis positive reports (high sensitivity) and to extrapolate the potential chart review savings from filtering

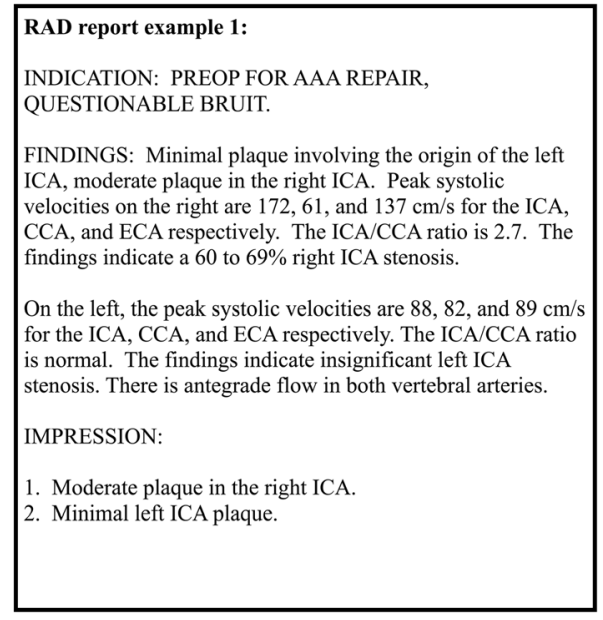

\begin{tabular}{l}
\hline TIU report example 2: \\
SUBJECTIVE: “Feels better than yesterday" \\
OBJECTIVE: vital signs, labs, and tests within normal range \\
ASSESSMENT: Non-significant ICA stenosis \\
PLAN: Follow up for flu shot next week. \\
\hline TIU report example 3: \\
PROCEDURE: Carotid Duplex. \\
INDICATIONS: Hx of ischemic stroke \\
FINDINGS: mild plaque in the carotid system bilaterally; \\
however, velocities are normal. Vertebral arteries demonstrate \\
antegrade flow. \\
IMPRESSION: Bilateral 10-20\% stenosis in the ICAs; not \\
hemodynamically significant.
\end{tabular}

Fig. 1 Sample texts by report type. Each text contains fictional, but realistic information 
stenosis negative reports (high negative predictive value). The dataset was randomly split into two sets: 200 development reports (100 RAD and 100 TIU notes) for algorithm knowledge base development [18] and 996 testing reports (498 RAD and 498 TIU notes) for information content analysis and algorithm evaluation. For the information content analysis, three research associates (domain experts) each independently and manually annotated the dataset for Sections, structures, and expressions as well as classified the report at the document-level as stenosis positive (if the report contained one or more mention of significant carotid stenosis) or stenosis negative (if the report contained only mentions of no/insignificant carotid stenosis). For the algorithm evaluation, the RAD reports were extracted from the VA EHR as two separate parts, Findings and Impressions. For the TIU reports, we parsed the Findings and Impressions using regular expressions written as a python script. We assessed pyConText's performance when provided the Findings only, Impressions only, and the full report.

\section{Information content assessment}

We aimed to characterize mentions of carotid stenosis findings according to Sections, structures, and expression types. Each report could have zero, one, or more relevant carotid stenosis findings recorded with zero, one, or more Sections, structures, and expression types.

\section{Sections}

RAD and TIU reports can be structured using canonical sections e.g., Indication, Findings, and Impression sections. We evaluated information content in the Findings (including Comments) versus Impressions (including Interpretations and Conclusions) sections [19].

\section{Structures}

VA notes can be generated using narrative or boilerplate templates in which the contents are saved as unstructured or semi-structured texts, respectively. For example, findings may be present in a variety of structures including: prose, lists, tables, headings, and other (Table 1). We

Table 1 Structure types with example sentences

\begin{tabular}{ll}
\hline & Example sentence \\
\hline Prose & "30-45 \% stenosis in the right ICA." \\
List & "1. Both ICAs are occluded." \\
Table & "95\% RICA $50 \%$ LICA $75 \%$ LECA" \\
Heading & "Right: ICA: stenosis $>70 \% "$ \\
Other & Any structures not listed above \\
\hline
\end{tabular}

evaluated information content according to these structure types [20].

\section{Expressions}

We have identified three types of expressions describing carotid stenosis findings: category, range, or exact. We characterized the information content according to these expression types [21] (Table 2).

\section{pyConText algorithm}

pyConText is a regular expression-based and rule-based system that extends the NegEx [22] and ConText [23] algorithms. NLP developers can train pyConText to identify critical findings and their contexts by defining regular expressions for these targeted findings and their desired modifiers within its knowledge base, respectively [24]. These modifiers can be used to filter spurious finding mentions that would otherwise generate false positives if generating a cohort based on simple keyword search. For example, a negation modifier can reduce false positives by filtering denied findings e.g., "no carotid stenosis". Furthermore, a severity modifier may reduce false positives by filtering insignificant findings e.g., "slight carotid stenosis". In a previous study, pyConText identified pulmonary embolism from computed tomography pulmonary angiograms by filtering spurious mentions using modifiers of certainty, temporality, and quality with high sensitivity (98\%) and positive predictive value $(83 \%)$. The pyConText pipeline is composed of three main parts: named entity recognition, assertion detection, and document-level classification.

\section{Named entity recognition and assertion detection}

Specifically, we adapted pyConText's knowledge base of findings and modifiers to filter no/insignificant carotid stenosis findings using regular expressions. These expressions contain "lexical variants" including synonyms, acronyms, abbreviations, and quantifications commonly documented in clinical text to represent carotid stenosis findings, semantic modifiers of severity, neurovascular anatomy, and sidedness, and linguistic modifiers of existence, temporality, and exam [25]. In Fig. 2, we provide the schema representing findings and each modifier as well as the possible normalized values. We represent these mentions and their normalized values using the following syntax: finding/modifier('lexical variant': normalized value). For example, in Fig. 3, "Moderate plaque

Table 2 Expression types with example sentences

\begin{tabular}{ll}
\hline & Example sentence \\
\hline Category & "severe stenosis" \\
Range & "stenosis ranging from 40 to $70 \% "$ \\
Exact & "60\% stenosis" \\
\hline
\end{tabular}




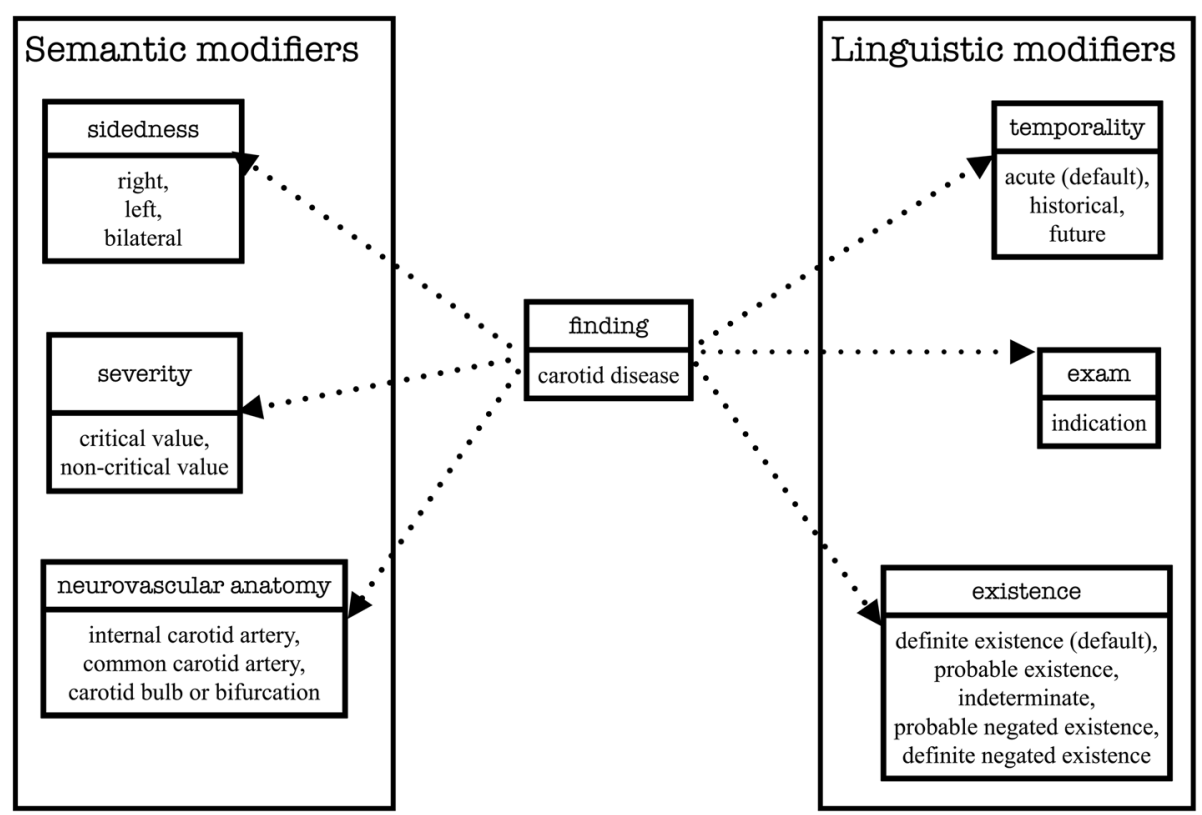

Fig. 2 Schema representing findings as well as semantic and linguistic modifiers and their possible normalized value sets

in the right ICA" is encoded as finding('plaque': carotid disease), severity('Moderate': critical value), neurovascular anatomy('ICA': internal carotid artery), sidedness('right': right), and existence(default: definite existence) using the knowledge base. pyConText leverages these normalized modifier values to determine whether a mention of a carotid finding(carotid disease) in the neurovascular anatomy(internal carotid artery, common carotid artery, carotid bulb or carotid bifurcation) represents no significant stenosis (stenosis with existence: definite negated existence), insignificant stenosis (stenosis with severity: non-critical value e.g., values less than $50 \%$ stenosis), or significant stenosis (stenosis with severity: critical values e.g., values equal or greater than $50 \%$ stenosis).

\section{Document classification}

For document-level classification, if either side or both sides of the internal or common carotid artery are determined to have significant stenosis, pyConText classifies the reports as stenosis positive; otherwise, it classifies it as stenosis negative. For RAD report example 1, in Fig. 3, the report would be classified as stenosis positive because two mentions of significant stenosis in the right internal carotid artery were identified. Figure 4 depicts RAD report example 1 fully processed by pyConText.

\section{pyConText evaluation}

pyConText applies a simple processing approach of segmenting and tokenizing sentences to process reports. The algorithm does not make use of Sections and structures. Therefore, we quantified how frequently complex document composition - Sections, structures, and expressions - are utilized to report carotid stenosis findings to gauge whether document decomposition processing such as section or structure tagging is needed to accurately extract findings. We evaluated the frequency of errors by Sections, structures, and expressions by comparing the predicted report classifications by pyConText to those generated by our domain experts.

Specifically, we defined a true positive when a report is correctly classified by pyConText as stenosis positive and a true negative when a report is correctly classified by pyConText as stenosis negative. In contrast, we defined a false positive when a report is spuriously classified by pyConText as stenosis positive and a false negative when a report is spuriously classified by pyConText as stenosis negative [12]. We assessed pyConText's performance by each Section and the full report using standard performance metrics of sensitivity, positive predictive value (PPV), specificity, and negative predictive value (NPV) as follows:

1. sensitivity $=\frac{\text { true positive }}{\text { true positive }+ \text { false negative }}$

2. positive predictive value $=$ hegative true positive

positive predictive value $=\underset{\text { true nesative }}{\text { true positive }+ \text { false positive }}$

3. specificity $=\frac{\text { true negative }}{\text { true negative }+ \text { false positive }}$

4. negative predictive value $=$

\section{Results}

Our testing set was comprised of 498 radiology reports (RAD) ultrasounds and 498 TIU notes. At the document-level, for RAD reports, 353 (71 \%) were stenosis negative and 145 (29\%) were stenosis positive; 


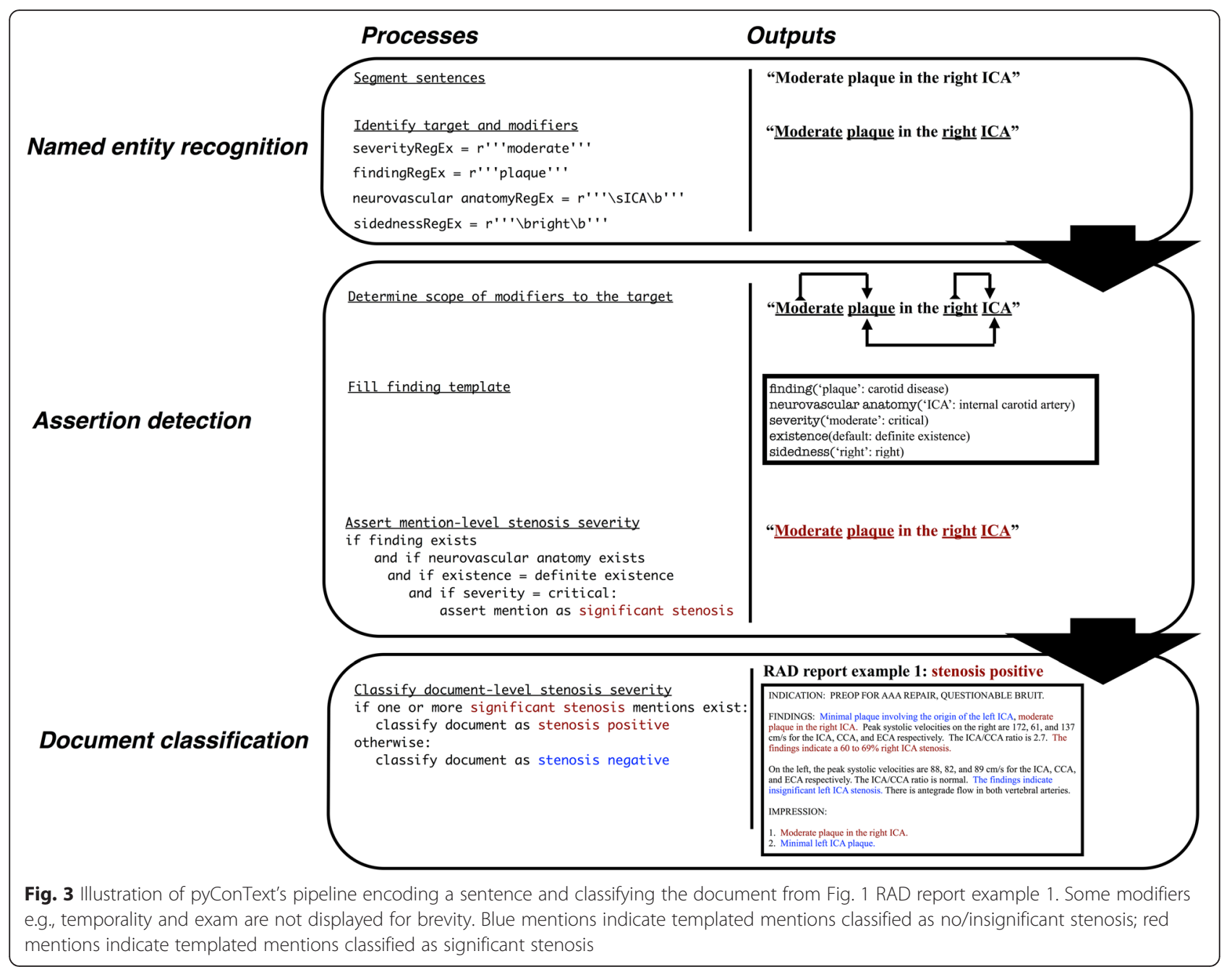

\section{RAD report example 1: stenosis positive \\ INDICATION: PREOP FOR AAA REPAIR, QUESTIONABLE BRUIT. \\ FINDINGS: Minimal plaque involving the origin of the left ICA, moderate plaque in the right ICA. Peak systolic velocities on the right are 172,61 , and $137 \mathrm{~cm} / \mathrm{s}$ for the ICA, CCA, and ECA respectively. The ICA/CCA ratio is 2.7 . The findings indicate a 60 to $69 \%$ right ICA stenosis. \\ On the left, the peak systolic velocities are 88,82 , and $89 \mathrm{~cm} / \mathrm{s}$ for the ICA, CCA, and ECA respectively. The ICA/CCA ratio is normal. The findings indicate insignificant left ICA stenosis. There is antegrade flow in both vertebral arteries. \\ IMPRESSION: \\ 1. Moderate plaque in the right ICA. \\ 2. Minimal left ICA plaque.}

Fig. 4 The resulting RAD report example 1 processed by pyConText from Fig. 3 for TIU reports, 396 (80 \%) were stenosis negative and 102 (20\%) were stenosis positive. The RAD training set distribution of $68 \%$ stenosis negative and $32 \%$ stenosis positive was comparable to the RAD testing set distribution. The TIU training set distribution of $87 \%$ stenosis negative and $13 \%$ stenosis positive reports differed slightly from the RAD testing set distribution.

\section{Information content assessment}

Of the 498 RAD reports, we observed most carotid mentions occur within the Impressions (488), are recorded using prose (706), and are expressed as categorical expressions (713). Carotid mentions occurred often within both Findings and Impressions (359) (Table 3). In contrast, of the 498 TIU reports, we observed that most carotid mentions did not occur in either the Findings or Impressions (286). However, similarly to RAD reports, carotid mentions were recorded using prose (294), and were expressed as categorical expressions (344) (Table 3). 
Table 3 According to report type, overall frequency of at least one carotid mention within sections, types of structures for all carotid mentions, and types of expressions for all carotid mentions

\begin{tabular}{llll}
\hline Information type & Information subtype & \multicolumn{2}{l}{ Report types } \\
\cline { 3 - 4 } & & RAD & TIU \\
\hline Sections & Findings Total & 368 & 106 \\
& Impressions Total & 488 & 173 \\
& Findings Only & 9 & 39 \\
& Impressions Only & 129 & 106 \\
& Both & 359 & 67 \\
& Neither/Not Applicable & 1 & 286 \\
Structures & & & \\
& Prose & 706 & 294 \\
& List & 256 & 76 \\
& Table & 0 & 36 \\
& Heading & 46 & 152 \\
& Other & 2 & 6 \\
& & & \\
Expressions & Category & 713 & 344 \\
& Range & 254 & 314 \\
& Exact & 48 & 19 \\
\hline
\end{tabular}

Findings Total $=$ Findings only + Both; Impressions Total = Impressions only + Both. Neither = report has Findings and Impressions, but does not contain carotid mentions; Not Applicable = report does not have Findings and Impressions

For RAD reports, within Findings, most carotid mentions were recorded as prose (306) followed by headings (66); within Impressions, most carotid mentions were recorded as prose (352) followed by lists (127) (Table 4). In contrast, for TIU reports, within Findings, most carotid mentions were recorded as headings (43) followed by tables (33); as Impressions, most carotid mentions were recorded as prose (88) followed by headings (48) (Table 4).

For RAD reports, of the carotid mentions reported within both Finding and Impression $(n=359$ reports; 379 paired mentions), there was repetition of structure types between sections (239 paired mentions, $63 \%$ )

Table 4 Structure type usage according to sections and report type

\begin{tabular}{llllll}
\hline & Prose & List & Table & Heading & Other \\
\hline RAD & & & & & \\
$\quad$ Findings & 306 & 3 & 0 & 66 & 3 \\
Impressions & 352 & 127 & 0 & 22 & 0 \\
TIU & & & & & \\
Findings & 25 & 6 & 33 & 43 & 0 \\
Impressions & 88 & 21 & 13 & 48 & 0 \\
\hline
\end{tabular}

Table 5 Structure type usage between Findings (rows) and Impressions (columns) for repetitive mentions by report type

\begin{tabular}{lcclll}
\hline & Prose & List & Table & Heading & Other \\
\hline RAD & & & & & \\
Prose & $233(61 \%)$ & $73(19 \%)$ & $0(0 \%)$ & $1(<1 \%)$ & $0(0 \%)$ \\
List & $1(<1 \%)$ & $1(<1 \%)$ & $0(0 \%)$ & $0(0 \%)$ & $0(0 \%)$ \\
Table & $0(0 \%)$ & $0(0 \%)$ & $0(0 \%)$ & $0(0 \%)$ & $0(0 \%)$ \\
Heading & $35(9 \%)$ & $27(7 \%)$ & $0(0 \%)$ & $5(1 \%)$ & $0(0 \%)$ \\
Other & $2(<1 \%)$ & $1(<1 \%)$ & $0(0 \%)$ & $0(0 \%)$ & $0(0 \%)$ \\
TIU & & & & & \\
Prose & $12(23 \%)$ & $4(7 \%)$ & $0(0 \%)$ & $3(6 \%)$ & $0(0 \%)$ \\
List & $0(0 \%)$ & $0(0 \%)$ & $0(0 \%)$ & $0(0 \%)$ & $0(0 \%)$ \\
Table & $15(28 \%)$ & $0(0 \%)$ & $1(2 \%)$ & $0(0 \%)$ & $0(0 \%)$ \\
Heading & $0(0 \%)$ & $9(17 \%)$ & $0(0 \%)$ & $9(17 \%)$ & $0(0 \%)$ \\
Other & $0(0 \%)$ & $0(0 \%)$ & $0(0 \%)$ & $0(0 \%)$ & $0(0 \%)$ \\
\hline
\end{tabular}

(diagonals in Table 5). In cases where a different structure was used between sections (140 paired mentions, $37 \%)$, the most frequent cases were Finding: prose/Impression: list, and Finding: heading/Impression: prose (discordants in Table 5). For TIU reports, of the carotid mentions reported within both Finding and Impression ( $n=67$ reports; 53 paired mentions), there was repetition of structure types between sections (22 paired mentions, $41 \%$ ) (diagonals in Table 5). In cases where a different structure was used between sections (31 paired mentions, $59 \%)$, the most frequent cases were Finding: table/Impression: prose followed by Finding: heading/Impression: list and Finding: heading/Impression: heading (discordants in Table 5).

For RAD reports, both Findings and Impressions, most carotid mentions were expressed as category (330 and 381, respectively) followed by range (73 and 178, respectively) (Table 6). We observed similar trends for TIU reports: category (73 and 116, respectively) followed by range (59 and 110, respectively) (Table 6).

For RAD reports, of the carotid mentions reported within both Findings and Impressions $(n=359$ reports; 526 paired mentions), there was repetition of expression types between sections (345 paired mentions, $66 \%$ ) (diagonals in Table 7). In the cases where a different expression type was used between sections (181 paired

Table 6 Expression type usage by sections and report type

\begin{tabular}{llll}
\hline & Category & Range & Exact \\
\hline RAD & & & \\
Findings & 330 & 73 & 25 \\
Impressions & 381 & 178 & 23 \\
TIU & & & \\
Findings & 73 & 59 & 8 \\
Impressions & 116 & 110 & 5 \\
\hline
\end{tabular}


Table 7 Expression type usage between Findings (rows) and Impressions (columns) for repetitive mentions by report type

\begin{tabular}{lccc}
\hline & Category & Range & Exact \\
\hline RAD & & & \\
Category & $278(53 \%)$ & $108(20 \%)$ & $14(3 \%)$ \\
Range & $35(7 \%)$ & $53(10 \%)$ & $2(<1 \%)$ \\
Exact & $16(3 \%)$ & $6(1 \%)$ & $14(3 \%)$ \\
TIU & & & \\
Category & $30(29 \%)$ & $23(22 \%)$ & $1(<1 \%)$ \\
Range & $26(25 \%)$ & $13(12 \%)$ & $3(3 \%)$ \\
Exact & $3(3 \%)$ & $4(4 \%)$ & $2(2 \%)$ \\
\hline
\end{tabular}

mentions, $34 \%)$, the most frequent cases were Finding: category/Impression: range and Finding: range/Impression: category (discordants in Table 7). For TIU reports, of the carotid finding mentions reported within both Findings and Impressions $(n=67$ reports; 105 paired mentions), there was repetition of expression types between sections (45 paired mentions, $43 \%$ ) (diagonals in Table 7). Similar to RAD reports, in the cases where a different expression type was used between sections (60 paired mentions, $57 \%$ ), the most frequent cases were Finding: category/Impression: range and Finding: range/Impression: category (discordants in Table 7).

\section{pyConText evaluation}

For RAD reports, pyConText achieved the highest positive predictive value (80\%) and specificity (93\%) when provided Impressions only (Table 8). However, the algorithm performed with lower sensitivity (74\%) and negative predictive value (90\%) compared to performance when provided the full report performing with higher sensitivity (88 \%) and negative predictive value (95\%). For TIU reports, we observed a similar trend. pyConText achieved the highest positive predictive value (76\%) and specificity (98\%) when provided Impressions only, but higher sensitivity (73\%) and negative predictive value (92\%) when provided the full report (Table 8 ).

Table $\mathbf{8}$ pyConText performance according to report type

\begin{tabular}{lllll}
\hline & Sensitivity & PPV & Specificity & NPV \\
\hline RAD & & & & \\
Findings & 57 & 67 & 88 & 83 \\
Impressions & 74 & $\mathbf{8 0}$ & $\mathbf{9 3}$ & 90 \\
Full report & $\mathbf{8 8}$ & 70 & 84 & $\mathbf{9 5}$ \\
TIU & & & & \\
Findings & 60 & 55 & 88 & 89 \\
Impressions & 19 & $\mathbf{7 6}$ & $\mathbf{9 8}$ & $\mathbf{9 2}$ \\
Full report & $\mathbf{7 3}$ & 58 & 87 & $\mathbf{9 2}$ \\
\hline
\end{tabular}

For each metric and report type, the highest metric value is bolded
For RAD reports, given the full report (including Findings and Impressions), pyConText generated 128 true and 56 false positive, and 297 true and 17 false negatives. The 73 reports were misclassified due to non-mutually exclusive errors of 96 prose, 42 list, 0 table, 12 headings, and 0 other. These non-mutually exclusive errors were the result of missed cues or erroneous scoping for 91 category, 50 range, and 16 exact expressions. In terms of locality of errors, 53 mentions were in both section types, 1 mention was in Findings only, 19 mentions were in Impressions only, and 0 mentions were in neither section. For TIU reports, given the full report (including Findings and Impressions), pyConText generated 74 true and 53 false positive, and 343 true and 28 false negatives. The 81 reports were misclassified due to non-mutually exclusive errors of 58 prose, 10 list, 8 table, 50 headings, and 0 others. These non-mutually exclusive errors were the result of missed cues or erroneous scoping for 74 category, 85 range, and 2 exact expressions. In terms of locality of errors, 14 mentions were in both sections, five mentions were in Findings only, 21 mentions were in Impressions only, and 41 mentions were in neither section.

\section{Discussion}

We conducted a pilot study evaluating information content of internal or common carotid finding mentions in terms of Section, structure, and expression usage. We also assessed pyConText's performance given these three factors.

\section{Information content assessment}

For RAD reports, most carotid mentions occurred in both Impressions and Findings with a substantial portion occurring in both sections. Overall mentions were recorded mainly as prose structure using category expressions. When carotid mentions were reported in Findings and Impressions, they were most often encoded in prose. For these cases, pyConText's simple text processing can accurately extract most of these mentions. In many cases, carotid mentions are repeated between Finding and Impressions, mainly as prose. In the case of discordant structure usage, this redundancy can be a processing advantage. Specifically, one of the most frequent cases was Finding: heading/Impression: prose. Therefore, if given the full report, pyConText can still correctly extract carotid mentions from the Impressions when it incorrectly extracts mentions from the Findings due to more complex structures like headings. Most mentions were found in Impressions composed mainly using expressions of category. In cases of repetitive descriptions between Findings and Impressions, most are Finding: category/Impression: category and mentions with discordant structure usage were Finding: category/Impression: range. These observations suggest 
that most severity descriptions can be extracted leveraging lexical, qualitative (e.g., "severe") regular expressions rather than quantitative (e.g., "70-99 \%") regular expressions.

For TIU reports, in contrast to RAD reports, most carotid mentions occurred in neither Findings nor Impressions, suggesting localized processing of reports for extracting carotid mentions would be suboptimal. In the few cases when carotid mentions were reported in Findings, they were most often headings followed by table structures. Similar to RAD reports, carotid mentions were reported in Impressions using prose, but also using headings, suggesting that complex document processing could be useful. Additionally, most mentions were found in Impressions composed mainly using expressions of category and exhibited the similar distributions of repetitive expression descriptions between Findings and Impressions.

For both RAD and TIU reports, we observed several mentions with two or more expressions or structures. For example, "55 \% moderate ICA stenosis" contains two expressions: exact (55\%) and category (moderate).

\section{pyConText evaluation}

We aimed to optimize the number of flagged positive cases for review (high sensitivity), while minimizing the loss of positive cases due to filtering (high negative predictive value); therefore, we conclude that pyConText performed best with the full report rather than with only the Finding or Impression sections. We hypothesize that providing pyConText with the full report resulted in the highest sensitivity because carotid mentions occurred with variable prevalence within Findings and Impressions (RAD) or within neither section type (TIU).

\section{Error analysis}

A detailed error analysis of pyConText's outputs revealed several areas of improvement to reduce false positives and negatives. For each error described, we provide an example and potential solution to boost performance within pyConText's processing pipeline.

Error 1: For both RAD and TIU reports, some false positives were due to missing category or range expressions for semantic modifiers. For instance, in Example 1, although we had "small" as a non-critical value for severity and "moderate" as a critical value for severity, we did not have "small to moderate" in our knowledge base due to mixing of quality (small) and quantity (moderate) descriptors. In these cases, our domain experts used the lower bound (small) to classify the severity value and assert the carotid mention as insignificant stenosis. However, pyConText did not recognize this as a range expression and the upper bound (moderate) was incorrectly used to classify the severity value and assert the finding as significant stenosis.

Example 1. "small to moderate amount of calcified plague in the left carotid bulb".

Potential solution 1: To improve assertion detection, we can add missed cues and expand upon existing regular expressions for the severity modifier. We could also add a rule that classifies ranges by the lowest bound for a severity value range by selecting the non-critical value over the critical value.

Error 2: In some cases, false positives were due to missing lexical variants for linguistic modifiers. In Example 2, we did not have a regular expression for "fails to demonstrate" for existence: definite negated existence; therefore, the algorithm classified the finding as significant stenosis.

Example 2. "examination of carotid arteries fails to demonstrate significant stenosis".

Potential solution 2: To improve assertion detection, again, we can add missed cues and expand upon existing regular expressions to identify linguistic modifiers from the text.

Error 3: Sometimes, the expressions were correct, but spuriously attributed to flow velocities that were not used to assert stenosis findings as in Example 3.

Example 3. "diameter reduction.. cca with velocity of 82.

Potential solution 3: To improve assertion detection and scope, we could have created another modifier velocity to correctly scope the severity modifier and filter this mention from classification.

Error 4: Our results suggest that we achieved lower performance for TIU reports than RAD reports due to more frequent usage of complex document structures such headings and tables rather than less complex document structures of prose and lists. In Example 4, "ICA" was correctly attributed to "Left $40 \%$ stenosis", but not associated to "Right $30 \%$ stenosis".

Example 4. "ICA: Left 40 \% stenosis." "Right $30 \%$ stenosis".

Potential solution 4: To improve assertion detection and scope, we could boost pyConText's performance by integrating outputs from a section tagger to identify mentions of neurovascular anatomy from headings/subheadings and associate them to all subsequent sentences within that section with relevant findings.

Error 5: In few examples, the algorithm generated a false negative due to its failure to identify co-referred findings of plaque. For Example 5, we observed two consecutive, long sentences. The first sentence contains a finding and neurovascular anatomy, but the second sentence contains its severity modifier. To link the severity in the second sentence to the finding and its neurovascular anatomy in the first sentence, we would 
need to resolve that the finding plaque in the second sentence co-refers to the finding plaque in the first sentence and merge their templates.

Example 5. "..calcified plaque in the left ica"..." “... data are consistent with between 50 and $80 \%$ stenosis by plaque".

Potential solution 5: To improve named entity recognition and assertion detection, we could handle coreference, by identifying co-referring expressions and either merging or resolving conflicting values for each finding template.

Error 6: Not all failures resulted in a document misclassification. In Example 6, the finding is not given, but implied by the checkbox and associated modifiers of sidedness, neurovascular anatomy, and severity so pyConText did not extract a stenosis finding. However, if this statement represented a significant stenosis mention, a false negative would have resulted.

Example 6. "Left ICA [x]: 0-15\%".

Potential solution 6: To improve named entity recognition and assertion detection, we could integrate outputs from document decomposition software [26] that readily identifies checkbox and question/answer constructs based on characters within the text. We could leverage these patterns to predict when and how these constructs should be used to extract assertions and correctly assert their scope when a finding is not explicitly mentioned.

Error 7: Similarly, although pyConText did not classify a finding mention in one sentence due to a missing modifier, it was able to identify and extract a finding mention from another sentence to correctly classify the report. In Example 7, pyConText does not find a neurovascular anatomy modifier for the second sentence, so it ignores it, but correctly classifies the report by correctly extracting information from the first sentence.

Example 7. "Right ICA occluded"... "1) occlusion on the right".

Potential solution 7: To improve document classification, we could classify sentences without a neurovascular anatomy modifier, but this strategy would have caused a significant increase in the number of false positives when the mention represents an irrelevant neurovascular anatomy such as the external carotid artery, increasing the number of reports for chart review by abstractors.

Error 8: Finally, false positives could be attributed to a lack of topical context. In Example 8, the sentence does not contain an actual finding, but rather guidelines for classifying mentions as significant stenosis.

Example 8. "definitions: 70-99 \% = significant stenosis"

Potential solution 8: To improve document classification, we could exclude extracted findings and assertions detected from all sentences that occur in the context of known guidelines e.g., documented NASCET legends by filtering these mention with a semantic modifier guidelines and regular expressions with guidelineassociated keywords like "definitions", "legend" or "NASCET".

Although many of these solutions could prove useful, they may add significantly to pyConText's processing time and complexity. For this study, it was only necessary to identify about 6,000 Veterans for cohort inclusion; therefore, we applied the system to the greater set of patient records based on these results. Because our goal is to retain as many stenosis positive cases as possible while filtering as many stenosis negative cases as possible, we provided pyConText the full report rather than only processing Impressions. To date, we have encoded over 150,000 RAD and 200,000 TIU reports. Given these results, we estimate that we have reduced the chart review task for study abstractors to about 85,000 $(\sim 25 \%)$ of the possible reports. The manual review of this filtered set was completed in 4 months by three abstractors rather than 12 months without the NLP filtering.

\section{Limitations}

Our study has a notable limitation. We only address reports from the VA EHR; therefore, pyConText's performance may or may not generalize to reports from other institutions. However, if the reports contain similar Sections, structures, and expressions, we would expect similar results. We will evaluate pyConText's generalizability on University of Utah Healthcare System reports for both genotype-phenotype association and stroke risk assessment studies in the near future.

\section{Future work}

Although for this study, we developed a sensitive NLP algorithm to identify high risk patients for stroke to support a comparative effectiveness review study, we plan to extend our algorithm to extract additional stroke risk factors for precise stroke subtype phenotyping e.g., ischemic and hemorrhagic stroke subtypes and endotypes e.g., ischemic stroke endotypes of cardiac embolism, large artery atherosclerosis, and lacunar infarction, other uncommon causes for genotype-phenotype association studies. We are actively generating a pipeline with our knowledge base authoring system, Knowledge Author, to leverage existing vocabularies such as the Unified Medical Language System (UMLS) [27] and Radiology Lexicon (RadLex) as well as ontologies such as our Modifier Ontology to encode these stroke risk factors in a more streamlined manner $[28,29]$.

\section{Conclusions}

We conclude that an information content analysis can provide important insights for algorithm development and evaluation including understanding information redundancy and challenges when processing clinical texts to 
identify stroke risk factors. Our study demonstrates that, in spite of these challenges, a simple NLP algorithm, can be leveraged to reduce chart review efforts by filtering reports with no/insignificant carotid stenosis findings and flagging reports with significant carotid stenosis findings from Veteran Health Administration clinical reports to support a comparative effectiveness study of stroke prevention strategies.

\section{Availability of the supporting data}

The supporting annotated dataset contains protected health information and is stored in the Veteran Affairs Informatics and Computing Infrastructure (VINCI). It is not available to researchers outside of the Department of Veteran Affairs. However, pyConText is available through https://github.com/chapmanbe/pyConTextNLP. Additional study information and collaborative development for pyConText can be found at http://toolfinder.chpc.utah.edu/ content/pycontext.

\begin{abstract}
Abbreviations
CPT: current procedural terminology; RAD: radiology; TIU: text integration utility; EHR: electronic health records; GWAS: genome-wide association studies; PheWAS: phenotype-wide association studies; ML: machine learning; NLP: natural language processing; eMERGE: electronic medical records and genomics; SHARPn: Strategic Health IT Research Program; PAD: peripheral artery disease; IRB: Institute Review Board; VA: veteran affairs; CHIR: consortium for healthcare informatics research; PPV: positive predictive value; NPV: negative predictive value; UMLS: unified medical language system; RadLex: radiology lexicon; VINCl: veteran affairs informatics and computing infrastructure; PMRP: personalized medicine research project; UIMA: unstructured information management architecture; QDM: quality data model; NIH: National Institute of Health.
\end{abstract}

\section{Competing interests}

We have no competing interests.

\section{Authors' contributions}

DM and BS designed the evaluation. EM and SK defined the stroke risk factor definition, queried the dataset from the Veteran Affairs electronic medical record, and facilitated the annotation of the dataset. BEC and WWC designed the original pyConText algorithm and knowledge base. DM extended these knowledge bases and adapted the pyConText algorithm. DM and MC wrote the original manuscript and provided this manuscript for editing and feedback to all coauthors. All authors read and approved the final manuscript.

\section{Acknowledgments}

This work was partially funded by VA HSR\&D Stroke QUERI RRP 12-185, NHLBI 1R01HL114563-01A1, NLM R01 LM010964, \& NIGMS R01GM090187. We thank Alexandra Woodbridge, Ann Abraham, Rosa Ahn, and Susan Saba for their excellent annotation efforts. We would also like to thank the Phenotype Day workshop organizing and program committee for the opportunity to extend our original submission [18] for this special issue and our anonymous reviewers for their insightful feedback.

\section{Author details}

${ }^{1}$ Department of Biomedical Informatics, University of Utah, Salt Lake City, UT, USA. ${ }^{2}$ IDEAS Center, Veteran Affair Health Care System, Salt Lake City, UT, USA. ${ }^{3}$ San Francisco Veteran Affair Health Care System, San Francisco, CA, USA.

Received: 21 October 2015 Accepted: 19 April 2016 Published online: 10 May 2016

\section{References}

1. Kullo IJ, Ding K, Jouni H, Smith CY, Chute CG. A genome-wide association study of red blood cell traits using the electronic medical record. PLOS ONE. 2010:5(9):e13011.

2. Denny JC, Ritchie MD, Basford MA, Pulley JM, Bastarache L, Brown-Gentry K, et al. PheWAS: demonstrating the feasibility of a phenome- wide scan to discover gene-disease associations. Bioinformatics. 2010;26(9):1205-10.

3. McCarty CA, Chisholm RL, Chute CG, et al. The eMERGE network: a consortium of biorepositories linked to electronic medical records data for conducting genomic studies. BMC Med Genet. 2011;4:13. doi:10.1186/1755-8794-4-13.

4. Kho AN, Hayes MG, Rasmussen-Torvik L, Pacheco JA, Thompson WK, Armstrong $\mathrm{AL}$, et al. Use of diverse electronic medical record systems to identify genetic risk for type 2 diabetes within a genome-wide association study. J Am Med Inform Assoc. 2012;19(2):212-8.

5. Kullo I, Fan J, Pathak J, Savova GK, Ali Z, Chute CG. Leveraging informatics for genetic studies: use of the electronic medical record to enable a genome-wide association study of peripheral arterial disease. J Am Med Inform Assoc. 2010;17(5):568-74.

6. Pathak J, Bailey KR, Beebe CE, Bethard S, Carrel DC, Chen P, et al. Normalization and standardization of electronic health records for high-throughput phenotyping: the SHARPn consortium. J Am Med Inform Assoc. 2013;20(e2):e341-8.

7. Shivade C, Raghavan P, Fosler-Lussier E, Embi PJ, Elhadad N, Johnson SB, et al. A review of approaches to identifying patient phenotype cohorts using electronic health records. J Am Med Inform Assoc. 2014;21(2):221-30.

8. Conway M, Berg RL, Carrell D, Denny J, Kho AN, Kullo IJ, et al. Analyzing the heterogeneity and complexity of electronic health record oriented phenotyping algorithms. AMIA Annual Symp. 2011;2011:274-83.

9. Hripcsak G, Albers D. Next-generation phenotyping of electronic health records. J Am Med Inform Assoc. 2013;20(1):117-21.

10. Brown DL, Morgenstern LB, Majersik JJ, Kleerekoper M, Lisabeth LD. Risk of fractures after stroke. Cerebrovasc Dis. 2008;25(1-2):95-9.

11. Birman-Deych E, Waterman AD, Yan Y, Nilasena DS, Radford MJ, Gage BF. Accuracy of ICD-9-CM codes for identifying cardiovascular and stroke risk factors. Med Care. 2005:43:480-5.

12. Hripcsak G, Rothschild AS. Agreement, the F-measure, and reliability in information retrieval. J Am Med Inform Assoc. 2005;12(3):296-8.

13. Savova GK, Fan J, Ye Z, Murphy SP, Zheng J, et al. Discovering peripheral arterial disease cases from radiology notes using natural language processing. AMIA Annual Symp. 2010;2010:722-6.

14. Wilke RA, Berg RL, Peissig P, Kitchner T, Sijercic B, McCarty CA, et al. Use of an electronic medical record for the identification of research subjects with diabetes mellitus. J Clin Med Res. 2007;5(1):1-7.

15. Garvin JH, DuVall SL, South BR, Bray B, Bolton D, Heavirland J, et al. Automated extraction of ejection fraction for quality measurement using regular expressions in Unstructured Information Management Architecture (UIMA) for heart failure. J Am Med Inform Assoc. 2012;19:859-66.

16. Thompson WK, Rasmussen LV, Pacheco JA, Peissig P, Denny JC, Kho AN, et al. An evaluation of the NQF quality data model for representing electronic health record driven phenotyping algorithms. AMIA Annual Symp. 2012; 2012:911-20.

17. Mowery DL, South BR, Garvin J, Franc D, Ashfaq S, Zamora T, Cheng E, Chapman BE, Keyhani S, Chapman WW. Adapting a natural language processing algorithm to support stroke cohort generation. HSR\&D/QUERI National Day. 2015.

18. Mowery DL, Chapman WW, Chapman BE, Conway M, South BE, Madden E, et al. Evaluating the Usage of Sections, Structures, and Expressions for Reporting and Extracting a Stroke Phenotype Risk Factor. Phenotype Day, Intelligent Systems for Molecular Biology. Dublin; 2015.

19. Gershanik EF, Lacson R, Khorasani R. Critical finding capture in the impression section of radiology reports. AMIA Annual Symp. 2011;2011:465-9.

20. South BR, Mowery DL, Tharp M, Carter M, Gundlapalli A, Vali M, et al. Extracting Social History and Functional Status from Veteran Affairs Clinical Documents. San Francisco: AMIA 2015 Joint Summits on Translational Science; 2015

21. Cheng EM, Bravata DM, El-Saden S, Vassar SD, Ofner S, Williams LS, et al. Carotid artery stenosis: wide variability in reporting formats-a review of 127 Veterans Affairs Medical Centers. Radiology. 2013;266(1):289-94.

22. Harkema H, Dowling JN, Thornblade T, Chapman WW. Context: an algorithm for determining negation, experiencer, and temporal status from clinical reports. J Biomed Inform. 2009;42(5):839-51. 
23. Chapman WW, Bridewell W, Hanbury P, Cooper GF, Buchanan BG. A simple algorithm for identifying negated findings and diseases in discharge summaries. J Biomed Inform. 2001;34:301-10.

24. Chapman B, Lee S, Kang H, Chapman WW. Document-level classification of $C T$ pulmonary angiography reports based on an extension of the ConText algorithm. J Biomed Inform. 2011;44:728-37.

25. Mowery DL, Franc D, Ashfaq S, Zamora T, Cheng E, Chapman WW, et al. Developing a knowledge base for detecting carotid stenosis with pyConText. Washington: AMIA Annual Symp; 2014. p. 1523.

26. Finch DK. Tagline: Information extraction for semi-structured text elements in medical progress notes. University of South Florida. Graduate Theses and Dissertations. 2012.

27. Bodenreider O. The Unified Medical Language System (UMLS): integrating biomedical terminology. Nucleic Acids Res. 2004;32:D267-70.

28. Scuba W, Tharp M, Tseytlin E, Liu Y, Drews FA, Chapman WW. Knowledge Author: creating domain content for NLP information extraction, Sixth International Symposium on Semantic Mining in Biomedicine. 2014. p. 99-103.

29. Scuba W, Tharp M, Mowery D, Tseytlin E, Liu Y, Drews FA, Chapman WW. Knowledge Author: facilitating user-driven, domain content development to support clinical information extraction. J Biomed Semantics. 2015. (accepted for publication).

Submit your next manuscript to BioMed Central and we will help you at every step:

- We accept pre-submission inquiries

- Our selector tool helps you to find the most relevant journal

- We provide round the clock customer support

- Convenient online submission

- Thorough peer review

- Inclusion in PubMed and all major indexing services

- Maximum visibility for your research

Submit your manuscript at www.biomedcentral.com/submit
Biomed Central 